La revue La revue pour l'histoire du CNRS

POUR L'HISTOIRE DU CNRS

$14 \mid 2006$

Le patrimoine scientifique

\title{
Histoire de la logique
}

Jean-Pierre Belna. Paris, Ellipses, Collection «L'esprit des sciences », 2005

Michel Blay

\section{OpenEdition}

Journals

Édition électronique

URL : https://journals.openedition.org/histoire-cnrs/473

DOI : $10.4000 /$ histoire-cnrs. 473

ISSN : 1955-2408

Éditeur

CNRS Éditions

Édition imprimée

Date de publication : 3 mai 2006

ISBN : 978-2-271-06350-2

ISSN : $1298-9800$

\section{Référence électronique}

Michel Blay, « Histoire de la logique », La revue pour l'histoire du CNRS [En ligne], 14 | 2006, mis en ligne le 07 mars 2006, consulté le 20 mai 2021. URL : http://journals.openedition.org/histoire-cnrs/473 DOI : https://doi.org/10.4000/histoire-cnrs.473

Ce document a été généré automatiquement le 20 mai 2021.

Comité pour l'histoire du CNRS 


\section{Histoire de la logique}

Jean-Pierre Belna. Paris, Ellipses, Collection «L'esprit des sciences », 2005

\section{Michel Blay}

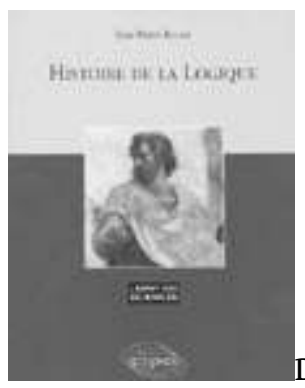

Dans son ouvrage intitulé Histoire de la logique, Jean-Pierre Belna se propose, sans faire appel à trop de connaissances techniques, de saisir, via son développement historique, ce " qu'est la logique ». Comme il le note dès les premières lignes de son introduction, vouloir en donner une définition simple ne va pas de soi : «Puisqu'il s'agit ici de raconter l'histoire de la logique, il est naturel de se demander ce qu'est la logique. La réponse est délicate. Les différents dictionnaires la définissent comme une science formelle, mettant en évidence soit le rapport à la vérité, soit à la déduction ou au raisonnement. Les auteurs d'un ouvrage récent d'initiation à la logique la présente comme «la science des lois du raisonnement, des règles de la pensée », qui «s'occupe de la forme des expressions » et « traite de la validité des raisonnements » ».

1 Suivant donc un plan historique, le livre de Jean-Pierre Belna est découpé en cinq chapitres couvrant, pour l'essentiel, l'histoire de la logique ou, plus précisément, délimitant ce qui, à travers le travail effectif des praticiens et des théoriciens, a permis la constitution sur la longue durée d'un corpus dénommé logique.

2 La logique apparaît en Grèce ; sa première mise en ordre est réalisée par Aristote. En cela elle s'inscrit dans le champ de la philosophie antique et, pour une large part, le structure. Jean-Pierre Belna analyse et présente très clairement cette logique en explicitant les procédés de la syllogistique tout en en montrant les limites. La logique antique est trop souvent réduite à l'œuvre aristotélicienne alors qu'une autre forme de la logique, la logique mégarico-stoïcienne, joue un rôle déterminant dans la 
constitution du champ de la logique en ce sens qu'elle est plutôt une logique des propositions, celle d'Aristote étant plutôt une logique des termes et qu'elle s'attache de plus à expliciter ses hypothèses. Tout cela est fort bien présenté et développé par JeanPierre Belna. Il conclut avec ses mots cette période très féconde : « La Grèce a inventé la logique, mais sous le signe d'un lien étroit avec le grammaire des langues naturelles. Malgré ses limites, la logique aristotélicienne est demeurée longtemps la référence des logiciens, tandis que celle des mégarico-stoïciens, pourtant plus fine sur bien des points, sombrait en grande partie dans l'oubli ».

3 L'époque médiévale reprend la logique aristotélicienne en la développant et en en précisant la pratique. Les médiévaux, en particulier, comme le montre Jean-Pierre Belna, ont attiré l'attention sur les théories des suppositions et des conséquences, sur l'énoncé de certaines lois de la logique propositionnelle et sur l'étude des paradoxes. Cette logique, la logique dite scolastique, a été largement rejetée à l'âge classique. Les débuts de la science moderne s'inscrivent à la fois dans une réflexion sur la notion de méthode et sur le rôle prépondérant, du point de vue de la constitution des savoirs, des mathématiques. On ne peut cependant négliger les travaux de logique de Roberval, de Mariotte et de G. W. Leibniz, ni ceux, bien évidemment, de Pascal et de Port-Royal. La logique ou l'art de penser, publié en 1662, renoue avec les travaux de logique dans leur rapport avec le langage.

$4 \quad$ C'est au XIXe siècle que la logique moderne se constitue en ce sens que naît la logique symbolique, logique ancrée dans un rapport étroit avec la mathématique. Quelques noms jalonnent cette histoire : George Boole, Auguste de Morgan puis Gottlob Frege. Il s'agit d'une mathématisation de la logique dont les enjeux vont traverser les XIXe et XXe siècles ; ils vont alimenter de multiples débats portant tant sur la nature même de la logique que sur les fondements des mathématiques.

Dans ces débats, dont les modalités sont bien mises en place par Jean-Pierre Belna, surgissent de nouveaux questionnements qui enrichissent les champs des sciences humaines et de la philosophie contemporaine. La logique aujourd'hui plurielle (modale, floue, etc.) joue donc dans ces champs un rôle de plus en plus important, rôle dont il serait indispensable de soumettre un peu plus souvent les modalités à l'examen philosophique et critique.

Le livre de Jean-Pierre Belna constitue une belle introduction au champ de la logique et aux problèmes qu'il soulève ; chacun pourra y apprendre beaucoup.

\section{AUTEUR}

\section{MICHEL BLAY}

Directeur de recherche au CNRS, SYRTE 